Figure 1. Crude incidence of EAMs by treatment exposure in patients with AS using TNFi and without TNFi treatment ( $95 \%$ Cls included)

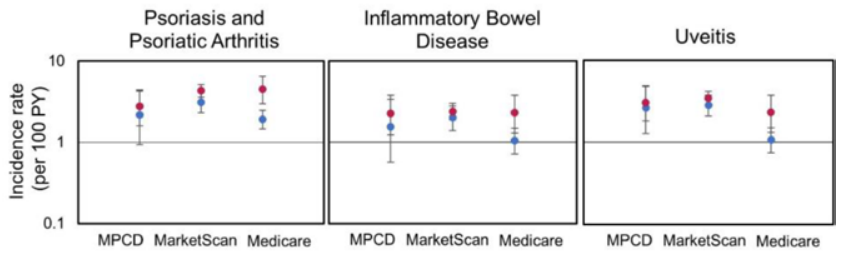

$\bullet$ TNFi $\bullet$ Without TNF

AS: ankylosing spondyloarthritis; $\mathrm{Cl}$ : confidence interval; EAM: extra-articular manifestation; MPCD: Multi-Payer Claims Database; PY: patient-years; TNFi: tumour necrosis factor inhibitor.

Figure 2. Propensity score-weighted hazard ratios of comorbidities and EAMs by treatment exposure in patients with $\mathrm{AS}$ using $\mathrm{TNFi}$ and without TNFi treatment

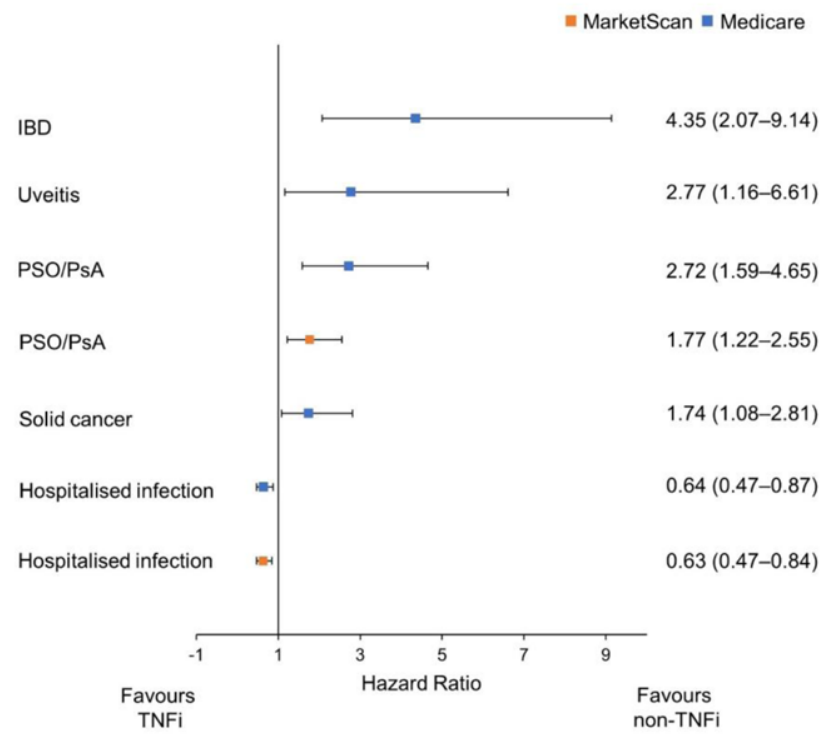

The forest plot shows propensity score-weighted hazard ratios and $95 \% \mathrm{Cls}$. Some data have been excluded due to a lack of events recorded in the dataset. All propensity scoreweighted hazard ratios with $\mathrm{Cls}$ crossing one were deemed non-significant and were therefore not included; this includes all relevant data from the MPCD database. AS: ankylosing spondylitis; CI: confidence interval; EAM: extra-articular manifestation; IBD: inflammatory bowel disease; MPCD: Multi-Payer Claims Database; PsA: psoriatic arthritis; PSO: psoriasis; TNFi: tumour necrosis factor inhibitor.

Acknowledgments: This study was funded by UCB Pharma. Editorial services were provided by Costello Medical.

Disclosure of Interests: Atul Deodhar Grant/research support from: AbbVie, Eli Lilly, GSK, Novartis, Pfizer, UCB, Consultant of: AbbVie, Amgen, Boehringer Ingelheim, Bristol Myer Squibb (BMS), Eli Lilly, GSK, Janssen, Novartis, Pfizer, UCB, Speakers bureau: AbbVie, Amgen, Boehringer Ingelheim, Bristol Myer Squibb (BMS), Eli Lilly, GSK, Janssen, Novartis, Pfizer, UCB, Kevin Winthrop Grant/research support from: Bristol-Myers Squibb, Consultant of: AbbVie, Bristol-Myers Squibb, Eli Lilly, Galapagos, Gilead, GSK, Pfizer Inc, Roche, UCB, Rhonda Bohn Consultant of: UCB Pharma, Benjamin Chan: None declared, Robert Suruki Employee of: UCB Pharma, Jeffrey Stark Employee of: UCB Pharma, Huifeng Yun Grant/research support from: Bristol-Myers Squibb and Pfizer, Sarah Siegel: None declared, Lang Chen: None declared, Jeffrey Curtis Grant/research support from: AbbVie, Amgen, Bristol-Myers Squibb, Corona, Crescendo, Genentech, Janssen, Pfizer, Roche and UCB Pharma, Consultant of: AbbVie, Amgen, Bristol-Myers Squibb, Corona, Crescendo, Genentech, Janssen, Pfizer, Roche and UCB Pharma

DOI: 10.1136/annrheumdis-2020-eular.4201

SAT0371

ARE ENGLISH-LANGUAGE VIDEOS ON YOUTUBE A USEFUL SOURCE OF INFORMATION FOR SPONDYLOARTHRITIS?

S. Elangovan ${ }^{1}$, Y. H. Kwan ${ }^{2}$, W. Fong ${ }^{1,3,4} .{ }^{1}$ Singapore General Hospital, Rheumatology and Immunology, Singapore, Singapore; ${ }^{2}$ Duke-NUS Medical
School, Program in Health Services and Systems Research, Singapore, Singapore; ${ }^{3}$ Duke-NUS Medical School, Singapore, Singapore; ${ }^{4}$ National University of Singapore, Yong Loo Lin School of Medicine, Singapore, Singapore

Background: Spondyloarthritis ( $\mathrm{SpA})$ is a family of chronic inflammatory disorders. Social media, such as YouTube, is a popular online platform where patients often visit for information. However, the validity of the content uploaded onto YouTube is not known.

Objectives: This study aimed to evaluate the content, reliability and quality of the most viewed English-language YouTube videos on SpA.

Methods: Keywords "spondyloarthritis", "spondyloarthropathy" and "ankylosing spondylitis" were searched on YouTube on October $7^{\text {th }}, 2019$. The top 270 videos were screened. Videos were excluded if they were irrelevant, in non-English language or if they had no audio. Total number of views, duration on YouTube (days), video length, upload date, number of likes, dislikes, subscribers and comments were recorded for videos. A modified 5-point DISCERN tool ${ }^{1}$ and the 5-point Global Quality Scale (GQS) score ${ }^{2}$ were used to assess the reliability and quality of the videos, with higher scores indicating greater reliability and quality respectively.

Results: Two hundred of 270 videos were included in the final analysis [ $61.5 \%$ from healthcare professionals, $37.0 \%$ from patients, $1.5 \%$ from news channels]. Of the 200 videos, 15 were uploaded within the last year and 112 in the last five years. $120(60 \%)$ were categorized as useful information (Group 1), $6(3 \%)$ as misleading information (Group 2), $52(26 \%)$ as useful patient opinion (Group 3 ) and $22(11 \%)$ as misleading patient opinion (Group 4). Useful videos were mainly from healthcare professionals or patients $(86 \%)$. Useful videos (Group 1 and 3) had higher median (IQR) number of subscribers [2700 (14700) vs 211 (457), $p<0.01$ ], reliability scores [3 (1) vs $2(1), p<0.01$ ] and GQS scores [3 (1) vs. 2 (1), p < 0.001] compared to misleading videos (Group 2 and 4), respectively.

Videos uploaded by healthcare professionals tended to have more useful information [94\% (116 of 123 ) vs. $66 \%$ (49 of 74 ), $p<0.001$ ] and had higher median (IQR) reliability scores [3 (1) vs $2(1), p<0.001]$ and GQS scores [3 (2) vs $2(1), p<0.001$ ] compared to patient uploaded videos respectively. Of the 5 (out of 123) videos from healthcare professionals that had misleading information, it was because of outdated information on diagnosis ( 3 videos) and treatment ( 5 videos) of SpA. Of the 22 videos that had misleading patient opinion, 9 (41\%) wrongly described the clinical features for SpA and $14(64 \%)$ portrayed the current evidence based treatment options as ineffective and described alternative treatment plans (i.e. diet restrictions, complementary and alternative medicine).

Conclusion: The majority of English language YouTube videos have useful information on the topic of SpA, however, $31 \%$ of patient opinions have inaccurate information on the clinical features and treatment options, and viewers need to be cognisant of these "fake news".

References:

[1] Charnock D, Shepperd S, Needham G, Gann R (1999) DISCERN: an instrument for judging the quality of written consumer health information on treatment choices. J Epidemiol Community Health 53(2): 105-111

[2] Bernard A, Langille M, Hughes S, Rose C, Leddin D, Veldhuyzen van Zanten $S$ (2007) A systematic review of patient inflammatory bowel disease information resources on the World Wide Web. Am J Gastroenterol 102(9):2070-2077 Disclosure of Interests: Sakktivel Elangovan: None declared, Yu Heng Kwan: None declared, Warren Fong Consultant of: Abbvie, Janssen, Novartis, Speakers bureau: Abbvie, Janssen, Novartis

DOI: 10.1136/annrheumdis-2020-eular.3109

\begin{tabular}{|l|l}
\hline SAT0372 & PATIENTS WITH PSORIATIC ARTHRITIS SHOW \\
HIGHER BONE DENSITY COMPARED TO AGE AND \\
GENDER MATCHED PATIENTS WITH ANKYLOSING \\
SPONDYLITIS
\end{tabular}

D. Freier ${ }^{1}$, E. Wiebe ${ }^{1}$, R. Biesen ${ }^{1}$, T. Buttgereit ${ }^{2}$, S. Hermann ${ }^{1}$, T. Gaber ${ }^{1}$, F. Buttgereit ${ }^{1}{ }^{1}$ Charité - Universitätsmedizin Berlin, Department of Rheumatology and Clinical Immunology, Berlin, Germany; ${ }^{2}$ CharitéUniversitätsmedizin Berlin, Department of Dermatology and Allergy, Berlin, Germany

Background: The prevalence of osteoporosis in inflammatory rheumatic diseases such as psoriatic arthritis (PsA) has not been sufficiently clarified yet, and the data in the literature are heterogeneous. In addition, it is still unclear to what extent patients with PsA differ in terms of bone density from patients with other forms of spondyloarthritis such as ankylosing spondylitis (AS).

Objectives: In an interim analysis of the Rh-GIOP Study (ClinicalTrials.gov Identifier NCT02719314), we observed that PsA patients demonstrated more frequently normal bone density than any other patient group analyzed (suffering from e.g. rheumatoid arthritis or systemic sclerosis). The main objective of this investigation was to compare bone density data from patients with PsA and 
AS, as both diseases belong to the spondyloarthritis group. 1100 patients with inflammatory rheumatic diseases provided the basis of Rh-GIOP, a prospective study monitoring glucocorticoid (GC)-induced osteoporosis in patients with rheumatic diseases. Rh-GIOP was established in 2015 at the Charité University Hospital. Bone mineral density data were measured by dual $\mathrm{x}$-ray absorptiometry (DXA).

Methods: 92 patients with PsA (65\% female) were compared with 51 patients suffering from AS (35\% female). Potential risk and protective factors (e.g. data on GC treatment, anti-rheumatic therapy), laboratory parameters (e.g. Vitamin D, alkaline phosphatase, calcium and inflammatory markers) and functional status (e.g. Health Assessment Questionnaire, sporting activities, back pain) were compared between these groups. Statistical analysis was performed descriptively using mean and standard deviation, t-tests for metric variables, and chi-square tests for nominal variables. Due to the heterogeneous gender distribution, an additional statistical matching was performed to compare patients matched by age and gender.

Results: Patients with PsA displayed significantly higher minimal T-scores than patients with AS $(p=0.003)$ even though patients with AS were younger and more often male $(p<0.001)$. AS patients showed a higher frequency of osteopenic bone densities $(p<0.05)$, however, no differences in the frequency of osteoporotic bone densities were found. Body-mass-index $(B M I)$ was significantly higher $(p<0.001)$ in PsA patients. PsA patients demonstrated a higher frequency of csDMARD use $(p<0.001)$. Additional analyses among PsA patients with and without csDMARDs revealed also significantly higher minimal T-scores in PsA patients taking csDMARDs ( $90 \%$ Methotrexate), and both groups showed the same average of age and gender distribution. Furthermore, AS patients complained significantly more often of back pain ( $96 \%$ vs. $74 \%, p=0.001$ ) than PsA patients. No differences in GC use or cumulative GC dose were found. All results could be confirmed when groups were matched by age and gender.

Conclusion: Our results demonstrate that patients with PsA display higher bone density compared to age and gender matched patients with ankylosing spondylitis. Possible influencing factors could be the higher frequency of csDMARD use, higher BMI or the lower frequency of back pain in PsA patients. Multivariate tests and additional biomarker investigations in larger cohorts are necessary to corroborate these findings and to identify underlying pathogenic differences which could serve for an explanation.

Disclosure of Interests: Desiree Freier: None declared, Edgar Wiebe: None declared, Robert Biesen: None declared, Thomas Buttgereit: None declared, Sandra Hermann: None declared, Timo Gaber: None declared, Frank Buttgereit Grant/research support from: Amgen, BMS, Celgene, Generic Assays, GSK, Hexal, Horizon, Lilly, medac, Mundipharma, Novartis, Pfizer, Roche, and Sanofi. DOI: 10.1136/annrheumdis-2020-eular.3566

\section{SAT0373 QUANTITATIVE ASSESSMENT OF RESPONSIVENESS IN SACROILIAC JOINTS MRI OF PATIENTS WITH AXIAL SPONDYLOARTHRITIS: A PILOT STUDY.}

C. Garrido-González¹, M. D. C. Castro Villegas², M. Ladehesa Pineda², J. L. Garrido-Castro ${ }^{1}$, R. Ortega Castro ${ }^{2}$, C. Gonzalez-Navas ${ }^{1}$, P. Zarco-Montejo ${ }^{3}$, R. Almodovar ${ }^{3}$, A. Bueno ${ }^{3}$, L. M. Molinero ${ }^{4}$, E. Collantes Estevez ${ }^{2}$ on behalf of COSPAR Study Group. ${ }^{1}$ Maimónides Biomedical Research Institute of Córdoba, Córdoba, Spain; ${ }^{2}$ Hospital Universitario Reina Sofia, Cordoba, Spain; ${ }^{3}$ Hospital Universitario Fundación Alcorcón, Madrid, Spain; ${ }^{4}$ ALCE Ingenieria, Madrid, Spain

Background: The presence of inflammatory signals in sacroiliac joints (SIJ), using MRI, is used for early diagnosis of axial spondyloarthritis (axSpA)[1] Some studies also demonstrate that this inflammation can be suppressed quite dramatically by TNF-a blockers. Different scoring methods to quantify inflammatory changes in SIJ using MRI have been defined and validated: SPARCC, Leeds, Berlin, and ASSpiMRI-a. However, its use is complex and subjective. Recently Zarco et al[2] developed a method to measure bone marrow edema (BME) in MRI images from SIJ. This method, in a semiautomatic procedure, allows to measure the area affected by inflammation and the signal intensity to produce an index: the SCAISS. A simplified version, the s-SCAISS, using only a semi-coronal slide, has been proposed with good validity and reliability results.

Objectives: To assess responsiveness of inflammation in SIJ of axSpA patients, treated with TNF-a inhibitors, using a novel score method: the s-SCAISS.

Methods: Two rheumatologists independently quantified SIJ images from axSpA patients by three methods (s-SCAISS, SPARCC and Berlin) on a single semi-coronal MRI slide (STIR). Patients were assessed before TNF-a therapy (PRE) and 3 months later (POST). Spearman correlations was used to analyze relationship between variables, Wilcoxon signed-rank test for significant differences and Cohen's d for calculating the effect size of improvement. Figure shows $\mathrm{MRI}$ images of a patient before and after treatment.
Results: 9 axSpA patients were recruited from the COSPAR cohort (44\% female, age $47 \pm 13$ years, disease duration $18 \pm 14$ years, BMI 29 \pm 4 ). Results PRE and POST are shown in Table: mean values (sd), statistical significance (NS, not significant; * $p<0.05 ;{ }^{* *}, p<0.01$ ), and Effect Size. In the first rows, differ ent scoring system for MRI inflammation appears: Area analyzed by s-SCAISS s-SCAISS, Berlin and SPARCC (using only a semi-coronal slide). Activity and functional indexes were lower with significant differences and a large effect size. Correlations of $\mathrm{s}$-SCAISS with Berlin (rho $=0.78 ; \mathrm{p}<0.05$ ) and SPARCC (rho=0.96; $p<0.001$ ) were good; with clinical disease activity outcomes were poor except with BASDAS (rho $=0.70 ; p<0.05$ ). The best correlation according improvements appeared comparing reduction of ASDAS with reduction of S-SCAISS (rho $=0.57$ ) but this difference was not significant. Although improvements in BASMI was not significant, a good correlation was found between improvement in s-SCAISS and BASMI (rho=-0.72; $<<0.05)$.

\begin{tabular}{lcccc}
\hline & PRE & POST & Sign & E.S. \\
\hline Area & $71.33(66.71)$ & $20.89(39.02)$ & $* \star$ & 0.86 -Large \\
s-SCAISS & $118.67(114.6)$ & $27.78(51.09)$ & $* \star$ & 0.98 -Large \\
BERLIN & $2.33(1.66)$ & $0.67(1.12)$ & $\star \star$ & 1.14 -Large \\
SPARCC & $3.11(2.26)$ & $0.78(1.39)$ & $* \star$ & 1.18 -Large \\
CRP & $13.1(9.4)$ & $4.1(3.2)$ & $* \star$ & 0.90 -Large \\
BASDAI & $6.5(1.7)$ & $4.4(2.7)$ & $*$ & 0.84 -Large \\
BASFI & $6.3(2.6)$ & $4.1(3.4)$ & $*$ & 0.69 -Medium \\
ASDAS & $3.6(1.0)$ & $2.3(1.2)$ & $\star \star$ & 1.15 -Large \\
BASDAS & $3.7(1.1)$ & $2.6(1.3)$ & $*$ & 0.96 -Large \\
BASMI & $3.6(1.5)$ & $3.3(1.3)$ & NS & \\
\hline
\end{tabular}

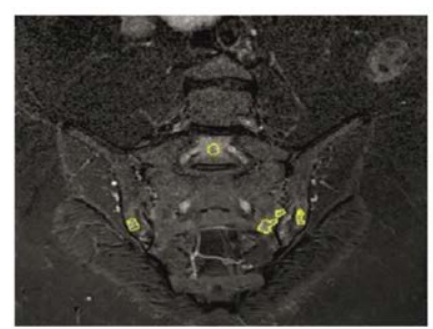

PRE: Area $=178 ;$ s-SCAISS $=234$ Berlin $=4 \quad$ Sparc $=7$

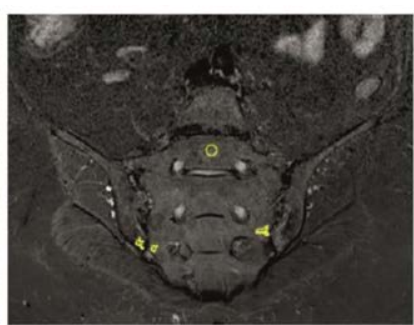
Berlin $=3 \quad$ Sparc $=$
POST: Area $=72 ;$ s-SCAISS $=100$

Conclusion: Different methods exist for quantifying inflammation in MRI images of SIJ in axSpA patients. According to our preliminary results, all of them had significant improvements in axSpA patients treated with anti tnf-a. The s-SCAISS index show good responsiveness, with similar features to validated indexes, but with an accuracy assessment of the BME area.

References:

[1] Defining active sacroiliitis on MRI for classification of axial spondyloarthritis: update by the ASAS MRI working group. Annals of the Rheumatic Diseases 2016;75:1958-1963.

[2] Development and validation of SCAISS, a tool for semi-automated quantification of sacroilitis by magnetic resonance in spondyloarthritis. Rheumatol Int. 2018 Oct;38(10):1919-1926.

Disclosure of Interests: Cristina Garrido-González: None declared, María del Carmen Castro Villegas: None declared, MLourdes Ladehesa Pineda: None declared, Juan L. Garrido-Castro: None declared, Rafaela Ortega Castro: None declared, Cristina Gonzalez-Navas: None declared, Pedro Zarco-Montejo Speakers bureau: Abbvie, MSD, Novartis, Pfizer., RAQUEL ALMODOVAR Speakers bureau: Abbvie, Celgene, Janssen, Lilly, Novartis, Pfizer.

, Angel Bueno: None declared, Luis Miguel Molinero: None declared, Eduardo Collantes Estevez Grant/research support from: ROCHE and Pfizer, Speakers bureau: ROCHE, Lilly, Bristol and Celgene

DOI: 10.1136/annrheumdis-2020-eular.2558

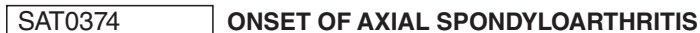 REPERCUSSIONS ON PATIENTS' SOCIAL AND FAMILY LIFE: RESULTS FROM THE EUROPEAN MAP OF AXIAL SPONDYLOARTHRITIS (EMAS)}

M. Garrido-Cumbrera ${ }^{1,2}$, V. Navarro-Compán ${ }^{3}$, C. Bundy ${ }^{4}$, R. Mahapatra ${ }^{5}$,

S. Makri' ${ }^{6}$, S. Sanz-Gómez ${ }^{1}$, L. Christen ${ }^{7}$, C. J. Delgado-Domínguez ${ }^{1}$,

D. Poddubnyy ${ }^{8,9}$ on behalf of EMAS Working Group. ${ }^{1}$ Health \& Territory

Research (HTR), Universidad de Sevilla, Sevilla, Spain; ${ }^{2}$ Spanish Federation

of Spondyloarthritis Associations, Madrid, Spain; ${ }^{3}$ IdiPAZ, University Hospital 